\title{
Anesthesiology: Is it a career, a job, or both?
}

\author{
Susan O'Leary, MD • Michael Murphy, MD
}

Received: 8 May 2015/Accepted: 12 June 2015/Published online: 19 June 2015

(c) Canadian Anesthesiologists' Society 2015

Anesthesia does not differ from other medical or surgical specialties when it entails subspecialization. We are experiencing burgeoning complexity in our patients, rapid changes in technology, and expanding medical evidence. These factors require anesthesiologists to consider what is needed in their practice not only to keep up with the curve but also to stay ahead of it. Centres compete for reputation in an increasingly competitive marketplace - a marketplace that is no longer simply local or regional but increasingly international. Furthermore, today's patients are medically savvy consumers with ever-increasing expectations. They have access to the best information, medical evidence, and centres of excellence. All these considerations are important as we strive as a specialty to deliver the highest quality care on an ever-expanding world stage.

We are fortunate in Canada to have a top notch education system for physicians and medical specialists. Following completion of a four-year medical degree and a five-year postgraduate residency training program, our graduates are preferred, and even sought after, as candidates for clinical subspecialty fellowship programs. The best of these programs develop subspecialists who are consummate clinicians, leading educators, and inquiring researchers.

S. O'Leary, MD ( $\square)$

Memorial University of Newfoundland, St. John's, NL, Canada

e-mail: susanoleary@bellaliant.net

M. Murphy, MD

University of Alberta, Edmonton, AB, Canada
The article by Khan et al. ${ }^{1}$ in this issue of the Journal is an interesting read. The authors have identified and quantified the various factors that motivate graduates of Canadian residency training programs to pursue clinical fellowship training. It is not surprising that critical care, cardiac anesthesia, and regional anesthesia are among the most popular subspecialties. The combination of acute care medicine and procedurally intense practices are compelling to many anesthesiologists. Nevertheless, skeptics may suggest that remuneration and other compensation might also influence the choice of fellowship. With most specialist anesthesiologists entering the workplace in their early to mid-thirties, female graduates face a slightly greater challenge balancing family and career. This might partly explain the authors' finding that male graduates are more likely to pursue a fellowship.

Khan et al. ${ }^{1}$ found that those pursuing additional training were motivated by three prime factors: personal interest, enhanced employability, and an interest in an academic career. These factors ring true to the authors of this editorial, as we have been involved in academic medicine our entire careers and have mentored dozens, if not hundreds, of residents on matters of career choice over those years.

The prevalent question in the minds of residents is whether additional training is 'worth it'. Coming to terms with that quandary requires individuals to reflect upon their own desires and interests and to consider those of family, both now and in the future. Many factors influence that decision, including the following (in no particular order):

- Is there a particular area of anesthesia that really engages my spirit?

- What is my perception of career and job?

- What is my notion of career satisfaction? 
- Do I want to end up in an academic centre?

- Where do I want to live?

- How do I best meet the many needs of my family?

- Do I enjoy the notion of helping to create the next generation of anesthesiologists?

- Am I interested in 'dancing' on a national or international stage?

- What do I want my legacy to be?

Ultimately, the decision depends on the individual's view of the time and effort it takes to become a subspecialist. Is it viewed as a price to pay to obtain a position in a centre of their choice or as an investment in a preferred career path?

We must not overlook the influence of the workforce marketplace on resident career choices. Over the years, there have been repeated discussions in numerous articles regarding anticipated shortages of anesthesiologists in Canada. As far back as 1970, an early editorial in this Journal $^{2}$ raised concerns over shortages of anesthesiologists and the future of anesthesia. The theme continued through to the 2000s with Dale Engen's report, "A demand-based assessment of the Canadian anesthesia workforce - 2002 through 2007". ${ }^{3}$ In 1995, however, Donen et al. ${ }^{4}$ previously questioned whether accurate Canadian anesthesia resource planning was possible given the numerous factors involved in the process. Indeed, it would now appear that the job market for recent Canadian anesthesiologists is significantly more competitive than it was during the past many years. This added factor must enter into a resident's decision to pursue additional training and should influence the advice we provide as senior colleagues and mentors.

Subspecialist clinical anesthesiologists are the backbone of academic departments of anesthesiology in Canada. Nevertheless, advanced expertise is needed in many areas of our specialty, including education, information systems, quality and safety, and leadership. Undoubtedly, residents completing residency training in Canada are accomplished clinicians and consultants on the same level as (and arguably superior to) those completing anesthesia training anywhere in the world. These graduates are superb clinical anesthesiologists who have skills as educators, are interested in discovery, possess an appetite for continuing competency, and have the potential to emerge as leaders in the specialty. The future of our specialty requires those of us in leadership roles to foster these directions and, in so doing, to encourage these kinds of fellowships and ensure that we both identify and mentor future leaders. The result will be a healthy future for our specialty that will allow our colleagues to maintain satisfying and productive jobs wherever their careers may lead.

\section{L'anesthésiologie: carrière, emploi, ou les deux?}

L'anesthésie n'est pas différente des autres spécialités médicales ou chirurgicales en matière de surspécialisation: nous assistons nous aussi à une complexification croissante de nos patients, à des changements technologiques rapides, et à une augmentation constante des données probantes. Pour ces diverses raisons, les anesthésiologistes doivent réfléchir à ce dont ils ont besoin dans leur pratique pour non seulement être à jour, mais également pour garder leur longueur d'avance. Les centres rivalisent pour leur réputation sur un marché de plus en plus compétitif - un marché qui n'est plus seulement local ou régional, mais de plus en plus international. Qui plus est, les patients d'aujourd'hui sont des consommateurs médicaux avisés et leurs attentes ne cessent de croître. Ils ont accès aux meilleurs renseignements, données probantes et centres d'excellence. Ces facteurs jouent tous un rôle important alors que nous nous efforçons, en tant que spécialité, d'offrir les soins de la plus haute qualité qui soit sur une scène mondiale en croissance perpétuelle.

Au Canada, les médecins et les spécialistes médicaux ont la chance de bénéficier d'un système d'éducation de pointe. Après l'obtention d'un diplôme médical qui achève quatre ans de formation et un programme postgrade de formation en résidence d'une durée de cinq ans, nos diplômés sont préférés, et même recherchés, en tant que candidats pour des programmes de stage ou 'fellowship' en surspécialisation clinique. Parmi ces programmes, les meilleurs forment des surspécialistes qui sont des cliniciens chevronnés, des éducateurs de pointe et des chercheurs curieux.

L'article de Khan et coll. ${ }^{1}$ dans ce numéro du Journal est intéressant. Les auteurs ont identifié et quantifié les divers facteurs qui motivent les diplômés des programmes de résidence canadiens à poursuivre une formation de surspécialisation clinique. Sans surprise, les soins critiques, l'anesthésie cardiaque et l'anesthésie régionale comptent parmi les surspécialités les plus courues. Une pratique combinant de la médecine des soins aigus et une importante variété de techniques est séduisante pour bon nombre d'anesthésiologistes. Les sceptiques suggéreront que le salaire et les autres formes de compensation pourraient également influencer le choix d'un fellowship. La plupart des anesthésiologistes spécialisés ne rejoignant le monde du travail que dans la trentaine ou plus tard, le défi pour les femmes diplômées est légèrement plus grand si elles souhaitent trouver un équilibre entre carrière et 
famille. Ceci pourrait expliquer en partie l'observation des auteurs selon laquelle les diplômés de sexe masculin ont davantage tendance à poursuivre une surspécialisation.

Khan et coll. ${ }^{1}$ ont observé que trois facteurs principaux motivaient les résidents à poursuivre une formation supplémentaire: l'intérêt personnel, une meilleure employabilité et un intérêt pour une carrière universitaire. Ces facteurs résonnent avec les auteurs de cet éditorial: en effet, nous sommes impliqués dans la médecine universitaire depuis les débuts de nos carrières et, au fil des années, nous avons servi de mentors pour des dizaines, voire des centaines de résidents en ce qui touche à leurs choix de carrière.

La question primordiale dans l'esprit des résidents est de savoir si une formation supplémentaire en «vaut la peine ». Pour pouvoir répondre à ce dilemme, les résidents doivent examiner leurs propres désirs et intérêts et tenir compte de ceux de leur famille, aujourd'hui et demain. De nombreux facteurs influencent cette décision, notamment (dans un ordre tout à fait aléatoire):

- Existe-t-il un domaine de l'anesthésie en particulier qui titille mon esprit?

- Comment est-ce que je perçois les notions de carrière et d'emploi?

- Qu'est-ce que j'entends par satisfaction professionnelle?

- Est-ce que je veux finir dans un centre académique?

- Où est-ce que je veux vivre?

- Comment répondre au mieux aux nombreux besoins de ma famille?

- Est-ce que j'aime l'idée d'aider à former la nouvelle génération d'anesthésiologistes?

- Est-ce que j'ai envie de 'jouer' sur une scène nationale et internationale?

- Qu'ai-je envie de laisser comme patrimoine?

En fin de compte, la décision repose sur la façon dont chacun perçoit le temps et les efforts nécessaires pour devenir un surspécialiste: est-ce un prix à payer pour obtenir un poste dans le centre de son choix, ou un investissement pour un parcours professionnel préféré?

Il est important de ne pas sous-estimer l'influence du marché du travail sur les choix de carrière des résidents. $\mathrm{Au}$ fil des années, plusieurs discussions ont été publiées dans de nombreux articles quant aux pénuries anticipées d'anesthésiologistes au Canada. En 1970 déjà, un des premiers éditoriaux du Journal ${ }^{2}$ émettait des inquiétudes quant aux pénuries d'anesthésiologistes et à l'avenir de l'anesthésie. Le thème s'est perpétué jusque dans les années 2000 avec le compte rendu de Dale Engen, « Évaluation des effectifs canadiens en anesthésie fondée sur la demande - de 2002 à 2007 ». En 1995 toutefois,
Donen $e t$ coll. ${ }^{4}$ s'étaient déjà posé la question de savoir s'il était possible de planifier de façon précise les ressources en anesthésie au Canada étant donné les nombreux facteurs impliqués dans ce processus. En effet, il semblerait que le marché du travail actuel soit considérablement plus compétitif pour les anesthésiologistes canadiens que par le passé. Le résident décidant de poursuivre une formation supplémentaire doit également tenir compte de ce facteur additionnel, qui devrait aussi influencer les conseils que nous prodiguons aux résidents en tant que collègues plus expérimentés et que mentors.

Les anesthésiologistes cliniques surspécialisés forment la colonne vertébrale des départements universitaires d'anesthésiologie au Canada. Cependant, nous avons besoin d'expertise dans plusieurs domaines de notre spécialité, notamment en éducation, en systèmes informatiques, en qualité et en sécurité, ainsi que dans le domaine du leadership. Il va sans dire que les résidents terminant leur résidence au Canada sont des cliniciens et des consultants accomplis au même niveau que (si ce n'est supérieurs à) les personnes terminant leur formation en anesthésie ailleurs dans le monde. Ces diplômés sont d'excellents anesthésiologistes cliniques qui possèdent des compétences d'éducateurs, sont curieux et souhaitent continuer à acquérir des compétences, et ont le potentiel de devenir des chefs de file de notre spécialité. Pour assurer l'avenir de notre spécialité, ceux d'entre nous en position de leadership doivent encourager ces orientations et ainsi favoriser ces types de fellowship afin de garantir que nous identifiions et formions les leaders de demain. Ainsi, notre spécialité profitera d'un avenir sain qui permettra à nos collègues de conserver des emplois satisfaisants et productifs, où que leurs carrières les mènent.

Conflicts of interest None declared.

Conflits d'intérêt aucun.

\section{References}

1. Khan J, Gilbert J, Sharma A, LeManach Y, Yee D. Perspectives of anesthesia residents training in Canada on fellowship training, research, and future practice location. Can J Anesth 2015; 62: this issue. DOI: 10.1007/s12630-015-0420-1.

2. Minuck M. Future manpower needs in anaesthetic practice. Can Anaesth Soc J 1970; 17: 1-3.

3. Engen DA, Morewood GH, Ghazar NJ, Ashbury $T$, VanDenKerkhof EG, Wang L. A demand-based assessment of the Canadian anesthesia workforce - 2002 through 2007. Can J Anesth 2005; 52: 18-25.

4. Donen N, White IW, Snidal L, Sanmartin CA. Canadian anaesthesia physician resource planning- is it possible? Can J Anaesth 1995; 42: 348-57. 